九州大学学術情報リポジトリ

Kyushu University Institutional Repository

Quantitative measurement of the phase locking of highly repetitive ultrashort optical pulses generated by a multifrequency continuous-wave Raman laser

Zaitsu, Shin-ichi

PRESTO, Japan Science and Technology Agency

Imasaka, Totaro

Division of Translational Research, Center for Future Chemistry, Kyushu University | Department of Applied Chemistry, Graduate School of Engineering, Kyushu University

http://hdl. hand le. net/2324/26423

出版情報: Applied Optics. 49 (9)，pp. 1586-1592，2010-03-20. Optical Society of America バージョン :

権利関係: (C) 2010 Optical Society of America 


\title{
Quantitative measurement of the phase locking of highly repetitive ultrashort optical pulses generated by a multifrequency continuous-wave Raman laser
}

\author{
Shin-ichi Zaitsu ${ }^{1,2, *}$ and Totaro Imasaka ${ }^{1,3}$ \\ 'Department of Applied Chemistry, Graduate School of Engineering, Kyushu University, \\ 744 Motooka, Nishi-ku, Fukuoka 819-0395, Japan \\ ${ }^{2}$ PRESTO, Japan Science and Technology Agency (JST), 4-1-8 Honcho Kawaguchi, \\ Saitama 332-0012, Japan \\ ${ }^{3}$ Division of Translational Research, Center for Future Chemistry, Kyushu University, \\ 744 Motooka, Nishi-ku, Fukuoka 819-0395, Japan \\ *Corresponding author: s-zaitsu@cstf.kyushu-u.ac.jp
}

Received 19 October 2009; accepted 26 January 2010; posted 17 February 2010 (Doc. ID 118742); published 11 March 2010

\begin{abstract}
We proposed and demonstrated a novel method for the evaluation of optical pulse trains generated by a multifrequency continuous-wave Raman laser operating at a mode separation of $17.6 \mathrm{THz}$. This approach is based on the detection of a nonlinear signal arising from the intensity modulation of a pulse train, which should provide a useful means for measuring the deviation from phase locking of multifrequency lasers. Our results suggest that an optimization of intracavity dispersion allows the generation of phaselocked multifrequency emissions, which leads to optical pulse trains at a repetition rate in excess of $10 \mathrm{THz}$. (C) 2010 Optical Society of America

OCIS codes: $\quad 120.5050,140.4050,140.3550$.
\end{abstract}

\section{Introduction}

The repetition rate of mode-locked lasers has been increased to $10 \mathrm{GHz}$ [1], which is associated with the evolution of optical-frequency comb technology. A light source with such a high repetition rate contributes directly to a broad range of ultrafast spectroscopic applications [2,3] and terahertz wave generation [4]. However, the configuration of the cavity currently used in mode-locked laser systems is no longer useful when the frequency of the repetition becomes larger than tens of terahertz, because a further increase in repetition rate is limited by a physical parameter, i.e., the length of the cavity. For instance, a $10 \mathrm{THz}$ mode-locked pulse train re-

0003-6935/10/091586-07\$15.00/0

(C) 2010 Optical Society of America quires a cavity length of no more than $15 \mu \mathrm{m}$. To overcome this problem, a harmonically mode-locked semiconductor laser was used and demonstrated a repetition rate of $2 \mathrm{THz}$ [5]. Alternative approaches, which are not based on the principle of the modelocked laser, have also been studied: beat-soliton conversion in a specially designed, highly nonlinear fiber [6], and a phase-locked system involving multiple semiconductor lasers [7]. The repetition rate obtained by these approaches, however, is limited to a few terahertz owing to the bandwidth of the key components (e.g., fibers and amplifiers). Hence, the future of mode-locked laser technology requires a novel idea for the generation of a pulse train with a higher repetition rate.

A phase-locked multifrequency continuous-wave (cw) Raman laser has been proposed and examined 
as a promising light source to generate an optical pulse train with a repetition rate in the terahertz region. It is predicted that phase-locked emission lines can be generated by stimulated Raman scattering and four-wave mixing in a Raman-active medium filled in a high-finesse optical cavity [8]. This type of laser would allow the production of an optical pulse train with a repetition rate of $>10 \mathrm{THz}$ because the repetition rate is determined by the Raman shift frequency, $\Omega$, of Raman-active molecules: orthohydrogen ( $Q(1), \Omega=125 \mathrm{THz} ; S(1), \Omega=17.6 \mathrm{THz})$; methane $(\Omega=87.5 \mathrm{THz})$; and deuterium $(Q(2), \Omega=$ $89.6 \mathrm{THz}$ ) [9]. We previously generated emission lines separated by $17.6 \mathrm{THz}$ using ortho-hydrogen as a Raman-active medium [10]. However, this multifrequency laser has not demonstrated phase locking because no method has been established to measure the phase relationship between emission lines separated by a frequency in the terahertz region.

Herein, we propose a method for the quantitative evaluation of phase locking for a multifrequency $\mathrm{cw}$ Raman laser that has the potential to generate a pulse train with a repetition rate exceeding $10 \mathrm{THz}$. This approach is based on the nonlinear demodulation of the modulated intensity of a pulse train using a detector with a quadratic response. This allows the quantification of a frequency deviation from the phase-locked state for a multifrequency emission separated by a frequency far beyond the response-time limits of a detector. In Section 2, we describe the theoretical background of the proposed method, including the basic concept of how to measure a mismatch of the separation frequency between adjacent modes and the effect of intracavity dispersion on the frequency mismatch. In Section 3, we present the experimental setup of the multifrequency cw Raman laser and systems for measurement of the output beam, i.e., the multifrequency emission separated by $17.6 \mathrm{THz}$ In Section 4 , we show autocorrelation traces reflecting the temporal waveform of optical pulse trains generated by the multifrequency $\mathrm{cw}$ Raman laser and the results obtained using the approach that we proposed in Section 2 . We discuss the present status of the phase relationship between the emission lines and specify the requirements for achievement of a phase-locking state. Finally, we summarize in Section 5 .

\section{Theoretical Background}

\section{A. Nonlinear Detection of Frequency Mismatch between} Longitudinal Modes

In the following discussion, in describing the phaselocked operation of a mode-locked laser, we only focus on three single-frequency longitudinal modes oscillating in a pumped cavity. Despite the small number of modes taken into account herein, the model can easily be extended to include more modes by choosing any three modes separated by a constant mode number. Figure 11 shows a series of longitudinal cavity modes including the three modes we focus

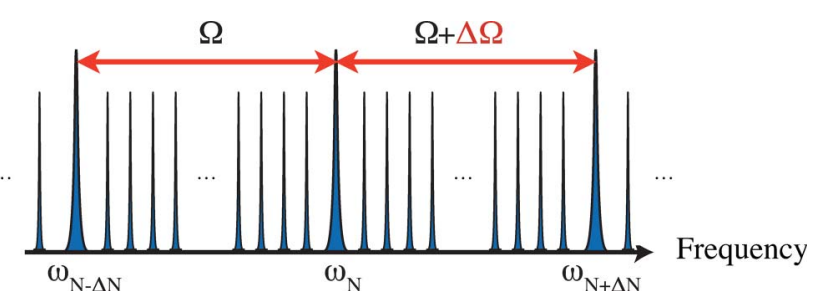

Fig. 1. (Color online) Three single-frequency longitudinal modes for describing the model discussed in this section. $\omega_{N-\Delta N}=\omega_{N}-\Omega$ and $\omega_{N+\Delta N}=\omega_{N}+\Omega+\Delta \Omega$. A subscript indicates the number of the mode.

on herein, $\omega_{N-\Delta N}, \omega_{N}$, and $\omega_{N+\Delta N}$, which are separated by a mode number, $\Delta N$. When the phases of these three modes are expressed as $\phi_{n}(t)=\omega_{n} t+$ $\phi_{n 0}$ for $n=N-\Delta N, N$, and $N+\Delta N$, where $\phi_{n 0}$ is the initial phase for each mode, a phase-locking condition for these modes requires that the following conditions be fulfilled at any moment:

$$
\phi_{N}(t)-\phi_{N-\Delta N}(t)=\phi_{N+\Delta N}(t)-\phi_{N}(t) .
$$

In the frequency domain, this is equivalent to the requirement that the separations between the adjacent modes be equal to one another: $\omega_{N}-\omega_{N-\Delta N}=\omega_{N+}$ $\Delta N-\omega_{N}$. Therefore, by defining $\Omega=\omega_{N}-\omega_{N-\Delta N}$ and $\Omega^{\prime}=\omega_{N+\Delta N}-\omega_{N}$, the phase-locked operation of a mode-locked laser can be achieved under the following conditions:

$$
\Delta \Omega=\Omega-\Omega^{\prime}=0 .
$$

That is, $\Delta \Omega=0$ ensures the generation of the phaselocked multifrequency emission that provides a pulse train at a repetition rate of $\Omega$. This indicates that a deviation from the phase-locked state of a modelocked laser can be determined quantitatively by a measurable parameter, $\Delta \Omega$. Here, we express the three modes as $E_{N-\Delta N}=\exp \left[-i\left(\omega_{N}-\Omega\right) t\right], E_{N}=\exp$ $\left[-i \omega_{N} t\right]$, and $E_{N+\Delta N}=\exp \left[-i\left(\omega_{N}+\Omega+\Delta \Omega\right) t\right]$. When a wave synthesized using these three emissions, $E(t)=E_{N-\Delta N}+E_{N}+E_{N+\Delta N}$, is detected by a linearresponse photodetector, the output signal from the detector is

$$
\begin{aligned}
I_{1}(t)= & E(t) E(t)^{*}=3+2 \cos \Omega t+2 \cos (\Omega+\Delta \Omega) t \\
& +2 \cos (2 \Omega+\Delta \Omega) t .
\end{aligned}
$$

When $\Omega$ is in the terahertz region, no information can be obtained for $\Delta \Omega$ from $I_{1}(t)$ because all the oscillation terms in Eq. (3) have frequencies larger than $\Omega$, which are far beyond the bandwidths of the currently available photodetectors. The effect of $\Delta \Omega$, however, appears as an intensity modulation at the frequency, $\Delta \Omega$, although this does not appear explicitly in Eq. (3). To derive this modulation effect from the signal, a nonlinear detector can be used in a manner analogous to that commonly used for demodulation of the amplitude in the radio-frequency range. When $E(t)$ is measured using a detector with a quadratic response, the output signal is 


$$
\begin{aligned}
I_{2}(t)= & {\left[E(t) E(t)^{*}\right]^{2}=15+4 \cos \Delta \Omega t+16 \cos \Omega t } \\
& +16 \cos (\Omega+\Delta \Omega) t+\cdots .
\end{aligned}
$$

In the above equation, the terms with frequencies larger than $2 \Omega$ are omitted. It should be emphasized that this equation includes a term oscillating at a frequency of $\Delta \Omega$ (the second term), allowing measurement of $\Delta \Omega$ using a nonlinear detector with a commonly available bandwidth. In addition, this term is independent of the frequency of the mode separation $\Omega$, which allows direct discrimination of the phase-locked state against the non-phase-locked state even for a pulse train with a terahertz repetition rate.

\section{B. Frequency Mismatch between Longitudinal Modes due} to Intracavity Dispersion

The $\Delta \Omega$ parameter, which is defined in the preceding subsection, does not generally vanish due to inevitable dispersion of the intracavity medium. Therefore, a mode-locked operation of the laser requires the control of intracavity dispersion, typically using a pair of prisms. The free spectral range (FSR) of the cavity containing a medium with a group refractive index, $n_{g}(\omega)$, is expressed as

$$
\Omega_{\mathrm{FSR}}(\omega)=\frac{c}{2 L \cdot n_{g}(\omega)},
$$

where $L$ is the length of the cavity and $c$ is the speed of light. The frequency dependence of $\Omega_{\mathrm{FSR}}(\omega)$ leads to unequally spaced longitudinal modes. Here, we again focus on the longitudinal modes, $\omega_{N-\Delta N}, \omega_{N}$, and $\omega_{N+\Delta N}$, as shown in Fig. 11. Since $\Omega_{\mathrm{FSR}}(\omega)$ generally increases with an increase in frequency in the optical region, Eq. (5) yields $\Delta \Omega>0$, preventing a mode-locked laser from operating in the phaselocked state that requires $\Delta \Omega=0$. The parameter $\Delta \Omega$ comprises two factors, $\Delta \Omega_{\text {medium }}$ arising from the dispersion in the intracavity medium and $\Delta \Omega_{\text {mirror }}$ from the dispersion of the cavity mirrors:

$$
\Delta \Omega=\Delta \Omega_{\text {medium }}+\Delta \Omega_{\text {mirror }} .
$$

$\Delta \Omega_{\text {medium }}$ is determined by summing the differences between FSRs for $\omega_{N+m}$ and $\omega_{N-m}$ :

$$
\Delta \Omega_{\text {medium }}=\frac{c}{2 L} \sum_{m=0}^{\Delta N-1}\left(\frac{1}{n_{g}\left(\omega_{N-m}\right)}-\frac{1}{n_{g}\left(\omega_{N+m}\right)}\right) .
$$

When designating the pressure of an intracavity gaseous medium as $p$ and using a valid approximation for a dilute gas, $n_{g}(\omega)=1+\alpha(\omega) p(1 \gg \alpha(\omega) p)$, we obtain

$$
\Delta \Omega_{\text {medium }} \approx \frac{c \cdot p}{2 L} \sum_{m=0}^{\Delta N-1}\left(\alpha\left(\omega_{N-m}\right)-\alpha\left(\omega_{N+m}\right)\right) .
$$

This equation shows that $\Delta \Omega_{\text {medium }}$ is proportional to the pressure of an intracavity medium when the cavity contains a low-pressure gaseous medium. Since
$\Delta \Omega_{\text {mirror }}$ is independent of the intracavity medium and is determined by the design of the mirror, $\Delta \Omega$ is expressed as a simple linear function of $p$.

\section{Experimental Setup}

A multifrequency cw Raman laser was first reported as a novel light source that enabled us to generate multiple emission lines separated by a frequency exceeding $10 \mathrm{THz}$ [8]. The main components of the laser system were a high-finesse cavity installed inside a chamber filled with a gaseous Raman-active medium and a single-frequency cw laser used as a pump source for the generation of high-order $\mathrm{cw}$ Raman emission lines. Multiple longitudinal modes separated by $>10 \mathrm{THz}$ oscillated simultaneously in the cavity with a high finesse in the wide spectral region $(>50 \mathrm{THz})$. In the previous study, we demonstrated the generation of multiple single-frequency emissions separated by $17.6 \mathrm{THz}$ on the basis of stimulated rotational Raman scattering of orthohydrogen [10]. In the present study, we again used a single-frequency Ti:sapphire laser (Coherent, MBR-110) as a pump source and a broadband high-finesse cavity (finesse $>10000,755-830 \mathrm{~nm}$ ), which were similar to those used in the previous experiment [see Fig. 2(a)]. To simplify the experiment, the cavity length was not stabilized automatically using a feedback loop, but instead was manually controlled by changing the voltage applied to a piezoactuator. This approach successfully maintained a resonant condition against the slight change in frequency of the pump laser for a period that was long enough to measure the output beam. This can be attributed to the self-stabilization caused by the heat deposition in the cavity [11].

The multifrequency emission from the cavity was measured using a photomultiplier tube (PMT) (Hamamatsu, 1P28) as a nonlinear detector for the operation principle described in the preceding paragraph. The Sb-Cs photocathode of this PMT has a two-photon response in the near-infrared region $(>650 \mathrm{~nm})$. Figure 2(b) shows the dependence of the PMT output voltage on the input power of the emission. Quadratic dependence on the input power is clearly shown even at power levels of less than $1 \mathrm{~mW}$. The PMT signal was fed to a spectrum analyzer (Advantest, U3741) to resolve the frequency components contained in the signal.

A temporal waveform of the output beam was measured using an interferometric autocorrelator equipped with a nonlinear detector that included a photocathode identical to that described in the preceding paragraph. This measurement was performed after passing the beam through a grating compressor to add group delay dispersion (GDD). This allowed investigation of the influence of an additional GDD on a temporal waveform. The spectrum of the beam was reshaped using an attenuation filter placed on the pathway of one of the spectral components before a folding mirror in the compressor. This spectrally reshaped beam was introduced into a Michelson 
interferometer with a collinear configuration, and the output beam from the interferometer was focused on the photocathode of the PMT using a lens with a $50 \mathrm{~mm}$ focal length. The beam was carefully aligned to irradiate the photocathode directly so as to avoid grids placed in front of the cathode. In the interferometer, a pair of one-side-coated beam splitters was placed in a back-to-back configuration to eliminate the asymmetric contribution of GDD arising from the substrates. This configuration allowed us to measure a temporal waveform in a broadband frequency range [12]. The length of one arm in the interferometer was slowly changed by translating one of the retroreflectors mounted on a piezo-controlled stage (translation distance, $100 \mu \mathrm{m}$ ), and the interferometric fringe (and an autocorrelation trace) was measured using an oscilloscope (Textronics, TDS1012).

\section{Results and Discussion}

First, we measured the temporal waveform of the multifrequency $\mathrm{cw}$ emission using the autocorrelator. The bottom graph in Fig. B(a) is the autocorrelation (AC) trace, at which two frequency components, i.e., the transmitted pump beam $(P, 773.6 \mathrm{~nm})$ and the first Stokes emission $(S 1,810.4 \mathrm{~nm})$, appeared in the spectrum [see the top graph in Fig. 3(a)]. The interval between the peaks in the modulated structure of the trace was $57 \mathrm{fs}$, which was identical to the multiplicative inverse of the frequency separation between the two emission lines, i.e., the rotational Raman shift frequency of ortho-hydrogen $(17.6 \mathrm{THz})$. Then, the cavity length was optimized in order to increase the intracavity power, which led to the generation of the second Stokes emission $(S 2,850.9 \mathrm{~nm})$ in addition to $P$ and $S 1$. Subsequently, the temporal waveform synthesized by these three singlefrequency emissions was measured after slightly attenuating the intensity of $P$ in order to obtain the spectrum with a nearly symmetrical shape with respect to $S 1$. Figure 3(b) shows the obtained spectrum (top) and the AC trace (bottom). The interval between the peaks ( $57 \mathrm{fs}$ ) was the same as that shown in Fig. 3(a). It should, however, be noted that the profile of the peak was narrower than that shown in Fig. 3(a). This was caused by the broadening of the total spectral width of the beam; i.e., three components were used for the synthesis of the peak. In our previous report [10], the peak in the AC trace had an asymmetric structure owing to the mismatch of GDD in the interferometer. On the other hand, the trace obtained herein clearly showed a symmetric structure with respect to the time of zero delay owing to a back-to-back configuration of the beam splitters in the autocorrelator. This result clearly indicates that an ultrafast temporal modulation of a cw emission can be achieved even at a frequency above $10 \mathrm{THz}$.

When the three emission lines consisting of $P, S 1$, and $S 2$ are phase locked, the synthesized waveform should provide a stable pulse train with a temporal

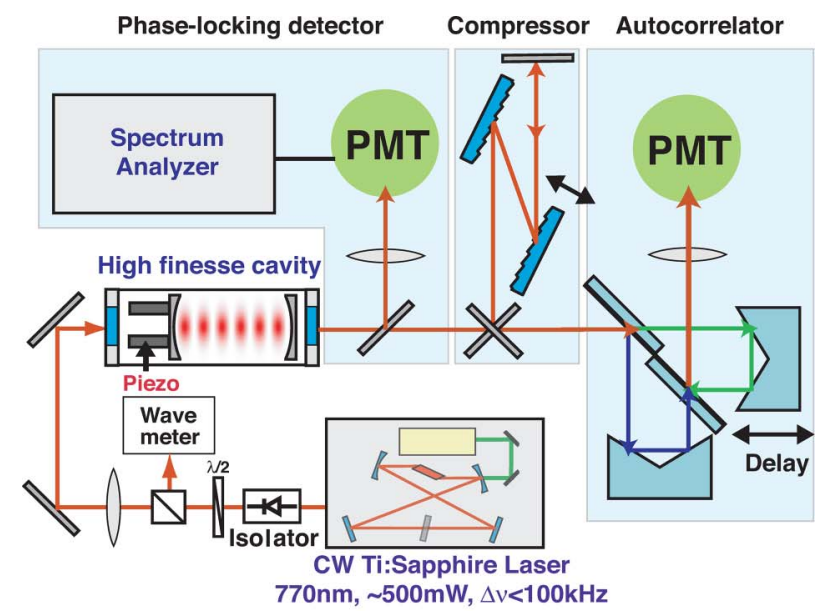

(a)

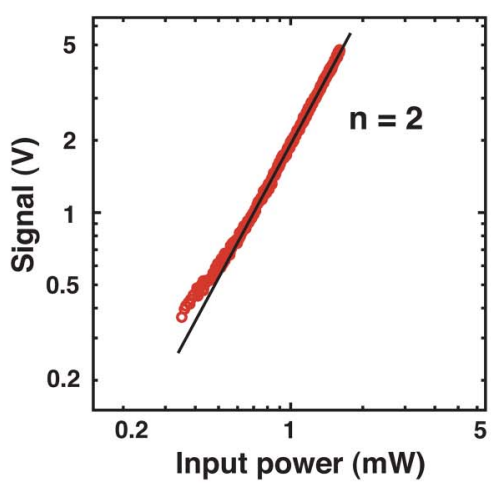

(b)

Fig. 2. (Color online) (a) Schematics of the experimental setup: the photomultiplier tube (PMT). The compressor consisted of a pair of gratings and a folding mirror. The autocorrelator consisted of a Michelson interferometer and a PMT used as a nonlinear detector. (b) Dependence of the signal intensity from the PMT on the input power of the laser. The $n$ parameter indicates the slope of the fitted line.

profile that does not change with time. On the other hand, when the phase difference between the emission lines varies depending on time, the situation is completely different. Herein, we define the difference between the phases as follows: $\Delta \phi(t)=2 \phi_{P}(t)-$ $\phi_{S 1}(t)-\phi_{S 2}(t)$, where $\phi_{P}(t), \phi_{S 1}(t)$, and $\phi_{S 2}(t)$ denote the phases for $P, S$, and $S 2$, respectively. Some simple algebra yields $\Delta \phi(t)=\Delta \Omega \cdot t$, which means the phase difference $\Delta \phi(t)$ results in periodic modulation of the intensity of the pulse train at a frequency of $\Delta \Omega$. The top graph in Fig. 4(a) shows the AC trace calculated from the spectrum shown in the top graph of Fig. 3(b) under the assumption of the phase locking; i.e., $\Delta \phi=0$. Although this trace was in good agreement with the trace shown in the bottom graph of Fig. $3(\mathrm{~b})$, this coincidence did not simply verify the generation of a phase-locked pulse train. This is because it takes longer than the period of the intensity modulation $(1 / \Delta \Omega)$ to measure an AC trace for a single pulse in a train of pulses. Thus, the effect of the unlocked 
phase, i.e., the fast modulation of the temporal waveform, was completely averaged and smeared in the AC trace.

To determine whether the multifrequency emission lines obtained herein were phase locked or not, the GDD was added to the beam because it critically affects a temporal waveform only for a phaselocked pulse train. When the terms higher than third order in a spectral phase are neglected, $\Delta \phi$ can be expressed as [13]

$$
\Delta \phi=\Omega^{2} \beta^{\prime \prime},
$$

where $\Omega$ is the frequency separation between adjacent emissions and $\beta^{\prime \prime}$ is the additional GDD. This indicates that $\Delta \phi=\pi$ is obtained for $\beta^{\prime \prime}=260 \mathrm{fs}^{2}$ at $\Omega=17.6 \mathrm{THz}$. The bottom graph in Fig. 4(a) shows the AC trace calculated from the observed spectrum [the top graph in Fig. 3(b)] for $\Delta \phi=\pi$ under the assumption of a phase-locked pulse. This graph clearly shows an apparent change in the shape against the AC trace shown at the top of Fig. 4(a). On the other hand, Fig. 4(b) shows the AC traces experimentally obtained before (top) and after (bottom) addition of GDD $(\Delta \phi=\pi)$ by changing the separation between the gratings placed before the autocorrelator. In contrast to Fig.4(a), no appreciable change was observed in the AC trace by the addition of GDD. This suggests that the phases of these three emission lines, $P, S 1$, and $S 2$, were not locked, at least under the present conditions. As discussed in Section 2, the phase unlocking among the modes of the multifrequency $\mathrm{cw}$ Raman laser arose from the dispersion of the intracavity medium. To produce a phase-locked pulse train, a quantitative understanding of the phase mismatch between the emission lines is indispensable;
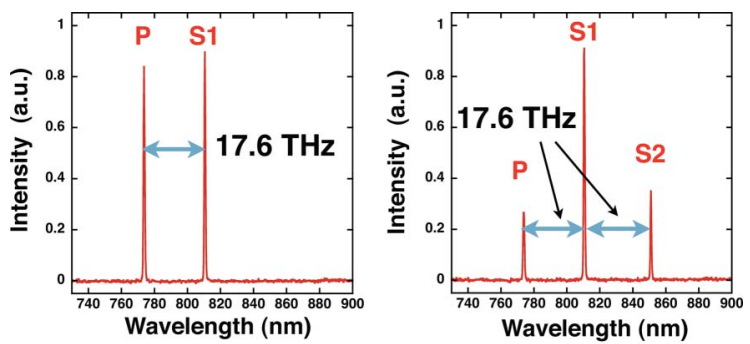

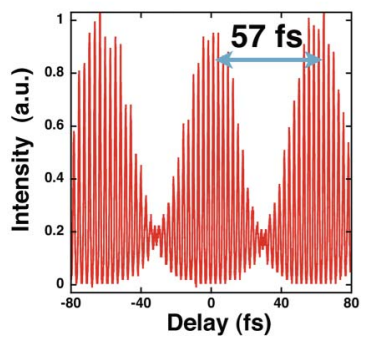

(a)

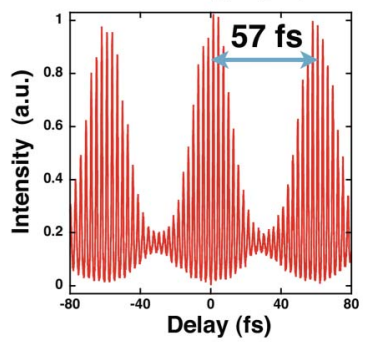

(b)
Fig. 3. (Color online) Spectra (top) and autocorrelation traces (bottom) of the multifrequency continuous-wave Raman laser. (a) The spectrum consisted of two frequency components, the pump $P$ and the first Stokes emission $S 1$. (b) The spectrum consisted of three frequency components, $P, S 1$, and the second Stokes emission $S 2$. nevertheless, the AC measurement was insufficient to provide us with information concerning modelocked operation.

To address this problem, a novel type of scheme described in Section 2 was used to quantitatively measure the deviation from the phase-locked state. Here, $\Delta \Omega$ was measured as a function of the hydrogen gas pressure from $0.1 \mathrm{MPa}$ to $0.9 \mathrm{MPa}$ with a step of $0.1 \mathrm{MPa}$. During the signal measurement, the generation of three emission lines was confirmed at each pressure. Figure 5(a) shows the spectrum of the signal obtained at a gas pressure of $0.4 \mathrm{MPa}$. The spectrum was comprised of a center peak at $23.15 \mathrm{MHz}$ and sidebands at a frequency separation of $c a$. $80 \mathrm{kHz}$. This frequency of separation corresponded to the frequency of intensity modulation of the measured beam [see an inset of Fig. 5(a)]. This intensity modulation was caused by a characteristic resonance in the servo system of the Ti:sapphire laser used as the pump beam; the frequency modulation was converted into the intensity modulation after passing the beam through the high-finesse cavity. Here, we concentrated on only the central peak in the signal at each pressure. The frequency of the measured peak is plotted in Fig. 5(b) as a function of the hydrogen gas pressure. The coefficient in front of $p$ in Eq. (8) was found to be $75 \mathrm{MHz} / \mathrm{MPa}$ on the basis of previously reported dispersion data of hydrogen gas [14]. Using this value and the assumption that $\Delta \Omega_{\text {mirror }}=-7.1 \mathrm{MHz}$, the measured points could be fitted with the linear dependence obtained from Eqs. (6) and (8). It should be noted that $\Delta \Omega=0$ was achieved at a pressure of $0.95 \mathrm{MPa}$; the beat signal and then the peak in Fig. 5(a) disappeared. This strongly suggests that the phase-locked pulse train was generated under this condition. In addition, at $\Delta \Omega=0$, the phase-matching condition was satisfied, (a) Calculation

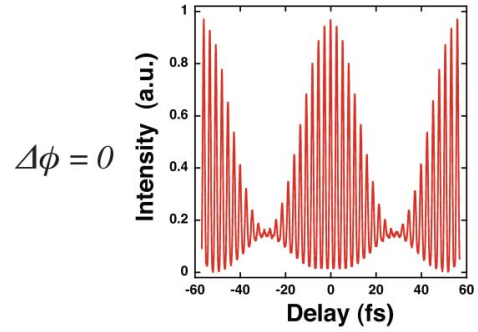

$\Delta \phi=\pi$

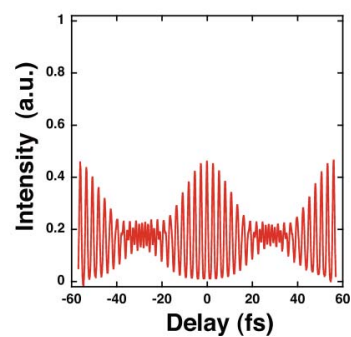

(b) Experiment
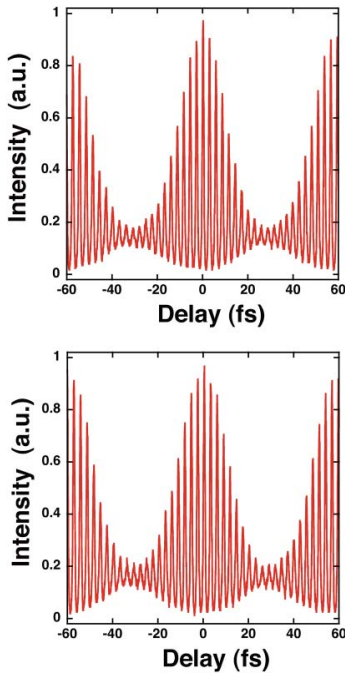

Fig. 4. (Color online) Autocorrelation traces obtained by (a) calculation and (b) measurement under assumptions of $\Delta \phi=0$ (top) and $\Delta \phi=\pi$ (bottom). 


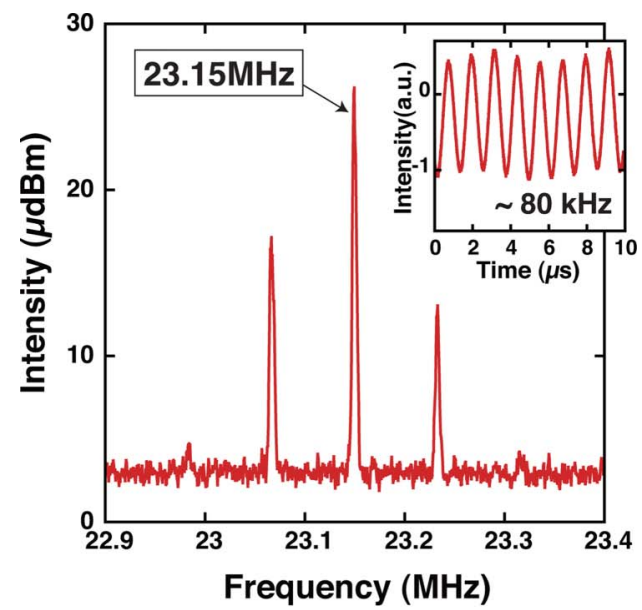

(a)

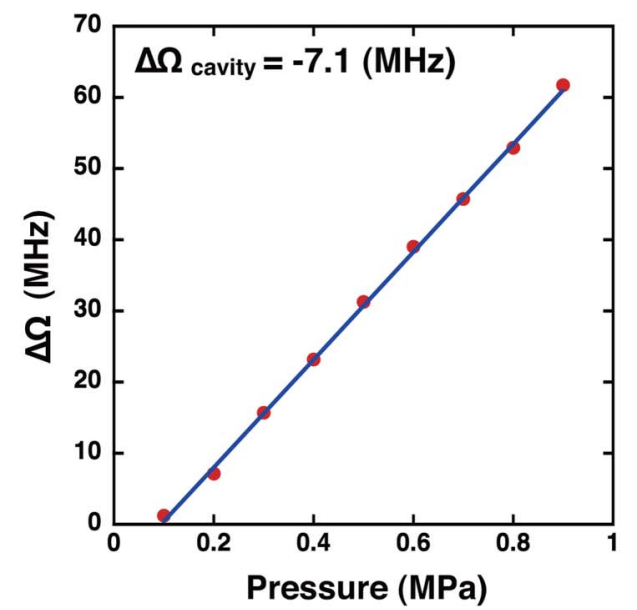

(b)

Fig. 5. (Color online) (a) Typical signal of the output beam (hydrogen pressure, 0.4 MPa). The inset shows the intensity modulation of the output beam. (b) Dependence of $\Delta \Omega$ on the hydrogen pressure. The points are fitted by $\Delta \Omega=75 p-7.1$.

and the process of an intracavity four-wave mixing was strongly enhanced [13]. This led to a strong coupling among the three emissions, $\omega_{p}, \omega_{s 1}$, and $\omega_{s 2}$, determining the frequency relationship between them as $\omega_{p}-\omega_{s 1}=\omega_{s 1}-\omega_{s 2}$; i.e., $\Delta \Omega=0$. Therefore, the phase-locked state of the multifrequency laser should be kept through the phase-matched four-wave mixing process when $\Delta \Omega=0$ is satisfied. It was previously reported that a highly-efficient phasematched four-wave mixing has been achieved in a high-finesse cavity with a negative dispersion [15]. These results suggest that the optimization of the intracavity dispersion will allow production of a phase-locked multifrequency emission $(\Delta \Omega=0)$ and generation of a train of highly stable optical pulses in excess of $10 \mathrm{THz}$.

\section{Conclusions}

In the present study, we quantitatively evaluated the deviation from the phase-locked state for an optical pulse train generated by a multifrequency cw Raman laser operating at a separation frequency of $17.6 \mathrm{THz}$. The approach used herein is based on the nonlinear demodulation of a beat signal caused by a phase mismatch between oscillating longitudinal modes. An autocorrelation measurement of the beam showed a pulselike structure modulated at $17.6 \mathrm{THz}$, and the dependence of the frequency deviation from the phase-locked state on the intracavity dispersion was in good agreement with the values calculated using the model taking into account the dispersion of the hydrogen gas. This result suggests that the phase-locked state of a multifrequency cw Raman laser can be achieved using a dispersion-controlled cavity to ensure a phase-matched intracavity fourwave mixing. This approach will lead to the generation of an optical pulse train with a repetition rate in excess of $10 \mathrm{THz}$.

This research was partially supported by the CASIO Science Promotion Foundation, the PRESTO program from Japan Science and Technology Agency (JST), Grants-in-Aid for Scientific Research, and the Global COE Program "Science for Future Molecular Systems" from the Ministry of Education, Culture, Science, Sports and Technology of Japan.

\section{References}

1. A. Bartels, D. Heinecke, and S. A. Diddams, "Passively mode-locked $10 \mathrm{GHz}$ femtosecond Ti:sapphire laser," Opt. Lett. 33, 1905-1907 (2008).

2. A. M. Weiner, D. E. Leaird, G. P. Wiederrecht, and K. A. Nelson, "Femtosecond pulse sequences used for optical manipulation of molecular motion," Science 247, 1317-1319 (1990).

3. A. Bartels, R. Cerna, C. Kistner, A. Thoma, F. Hudert, C. Janke, and T. Dekorsy, "Ultrafast time-domain spectroscopy based on high-speed asynchronous optical sampling," Rev. Sci. Instrum. 78, 035107 (2007).

4. Y. Sasaki, H. Yokoyama, and H. Ito, "Dual-wavelength opticalpulse source based on diode lasers for high-repetition-rate, narrow-bandwidth terahertz-wave generation," Opt. Express 12, 3066-3071 (2004).

5. D. A. Yanson, M. W. Street, S. D. McDougall, I. G. Thayne, and J. H. Marsh, "Terahertz repetition frequencies from harmonic mode-locked monolithic compound-cavity laser diodes," Appl. Phys. Lett. 78, 3571-3573 (2001).

6. Y. Ozeki, S. Takasaka, J. Hiroishi, R. Suguzaki, T. Yagi, M. Sakano, and S. Namiki, "Generation of $1 \mathrm{THz}$ repetition rate, $97 \mathrm{fs}$ optical pulse train based on comb-like profiled fibre," Electron. Lett. 41, 1048-1050 (2005).

7. M. Hyodo, K. S. Abedin, and N. Onodera, "Fourier synthesis of $1.8 \mathrm{THz}$ optical-pulse trains by phase locking of three independent semiconductor lasers," Opt. Lett. 26, 340-342 (2001).

8. K. Shinzen, Y. Hirakawa, and T. Imasaka, "Generation of highly repetitive optical pulses based on intracavity four-wave Raman mixing," Phys. Rev. Lett. 87, 223901 (2001).

9. D. C. Hanna, D. J. Pointer, and D. J. Pratt, "Stimulated Raman scattering of picosecond light pulses in hydrogen, deuterium, and methane," IEEE J. Quantum Electron. 22, 332-336 (1986).

10. S. Zaitsu, C. Eshima, K. Ihara, and T. Imasaka, "Generation of a continuous-wave pulse train at a repetition rate of 17.6 THz," J. Opt. Soc. Am. B 24, 1037-1041 (2007).

11. P. Dubé, L.-S. Ma, J. Ye, P. Jungner, and J. L. Hall, "Thermally induced self-locking of an optical cavity by overtone 
absorption in acetylene gas,” J. Opt. Soc. Am. B 13, 2041-2054 (1996).

12. C. Spielman, L. Xu, and F. Krausz, "Measurement of interferometric autocorrelations: comment," Appl. Opt. 36, 2523-2525 (1997).

13. G. P. Agrawal, Nonlinear Fiber Optics (Academic, 2001), Chap. 10.
14. E. R. Peck and S. Huang, "Refractivity and dispersion of hydrogen in the visible and near infrared," J. Opt. Soc. Am. 67, 1550-1554 (1977).

15. S. Zaitsu, H. Izaki, and T. Imasaka, "Phase-matched Raman-resonant four-wave mixing in a dispersioncompensated high-finesse optical cavity," Phys. Rev. Lett. 100, 073901 (2008). 